\title{
Invariant Object Representation and Recognition Using Lie Algebra of Perceptual Vector Fields
}

\author{
Jinhui Chao ${ }^{1}$, Akira Karasudani ${ }^{1}$ and Kenji Minowa ${ }^{1}$ \\ 1 Chuo University, 1-13-27, Kasuga, Bunkyo-ku, Tokyo, 112, Japan \\ 2 Tokyo Institute of Technology, 2-12-1, Ookayama, Meguro-ku, Tokyo, 152, Japan
}

\begin{abstract}
This paper presents a global method to represent objects invariantly under Euclidean motions using Lie algebra of perceptional vector field of the objects. We focus on the linear Lie sub-algebra of the tangent or normal Lie algebra of objects and use pure local information in these Lie algebra to represent global shapes. It is shown that this simple subalgebra can represent algebraic shapes and a much wider class of non-algebraic shapes as well. In this way, an occlusion-robust and fast recognition method is derived.
\end{abstract}

\section{Introduction}

Invariant 3D object representation is a fundamental issue in object recognition, CAD modeling, computer graphics and recently in object-based image coding. Most 3D object representation methods have been proposed so far in order to represent free 3D shapes more efficiently than polyhedron representations, based on extracting local or semi-local invariant features of objects from their planer intensity images or 3D range images for template matching[1][2].

A popular approach is to use point-wise differential invariant indexing, e.g. curvatures and torsions or the extended Gaussian maps. However, since the global shapes are represented as a loose collections of local features, e.g. the interpretation table, one has to verify a large number of hypotheses on the interpretation tree before reaching the correct matching.

A semi-local representation is to employ algebraic invariants of algebraic curves or surfaces, e.g. conics. However, since polynomial approximation is locally valid only, i.e., over small neighborhoods, a great deal of patches will often be required to represent a sculpted object. Thus, once again exhaustive search in a large interpretation table is necessary.

Thus it seems desirable to have certain global representations for objects. Unfortunately, it is unlikely that there could exist any finite-parameter models of such representations for arbitrary objects. Approximation models such as generalized cylinders or super-quadratics can be used. However, it is usually difficult to find the invariant features for such non-algebraic shapes, especially to find the complete set of invariants in order to uniquely determine the objects. Besides, a global representation also intends to be vulnerable to occlusion. 
The approaches to define invariants of objects with respect to the transformation groups as Lie groups seem attractive in that it may define global differential invariants based on local features[1] [2][3][4]. However, although these works take advantage of certain nice properties of the Euclidean transformations as Lie groups, they do not make any serious use of intrinsic properties of the objects themselves. Therefore, in order to obtain invariants for an objects, one has to solve a complicated PDE under each transformations. The task to find such general solution is in fact so difficult for arbitrary objects that until now it seems hard to obtain explicitly any truly new and nontrivial invariant by these approaches.

In this paper, we address ourselves to $3 \mathrm{D}$ objects as $2 \mathrm{D}$ smooth surfaces which can be represented by Lie groups.In particular, we introduce a structure of Lie algebra to the tangent and normal vector fields of the object surfaces, then we consider not only the transformation groups as Lie groups, which are the classical groups, but the Lie groups which define the objects themselves as well. A novel approach is proposed to represent the shapes as Lie groups that are generated by the tangential or normal vector fields on surfaces of the objects which are their Lie algebras. Then invariance of the objects as Lie groups is considered under the action of the classical groups on these Lie algebras. With this neat structure, we can make a full use of the powerful relationship between Lie groups and Lie algebras. e.g., it is known that the Lie group which we used to represent a global shape can be uniquely determined by purely local information in its Lie algebra.

Even in this case, however, it still seems to be nontrivial for arbitrary Lie groups to find a flexible representation whose complete set of invariants can be easily calculated. In this paper, we restrict ourselves to a subclasse of the Lie algebras that has simple representations, i.e., the linear Lie algebras, which we assume as the tangential or normal Lie algebras of the objects. The complete set of invariants can be easily calculated from image data by solving a system of linear equations. Their robustness against noises can be achieved by implementation of these recognition algorithms with the Hugh transform and the least mean square fitting method.

Features of the proposed method also include robustness against occlusion since theoretically the global shapes can be uniquely determined from more than three points in 2D cases and four points in 3D cases on any small part of the surfaces of the objects.

Also, these models extend a great deal of descriptive power of existing models for free shapes. In spite of the simplicity of these sub-algebras, we can cover enough wide classes of shapes, including algebraic shapes and non-algebraic ones.

\section{Object Representation by Linear Lie algebra of tangent and normal vector fields}

A Lie group $M$ is a smooth manifold on which smooth operations $(x, y) \mapsto x y$, for $x, y \in M$ and $x \mapsto x^{-1}$ are defined. It is known that a Lie group possesses, 
as its infinitesimal generator, a Lie algebra of the left-invariant tangential vector fields on it, which is closed for an operation called Lie bracket and satisfies an equation called Jacobi identity. In other words, Lie groups are homogeneous spaces or integral flows generated by the Lie algebras, or by the tangential vector fields on their surfaces.

We define the linear Lie subalgebra $\mathfrak{g}$ with an infinitesimal generator as

$$
\begin{aligned}
& \mathcal{L}=\left(\sum_{i=1}^{n} a_{1 i} x^{i}, \cdots, \sum_{i=1}^{n} a_{n i} x^{i}\right)\left(\begin{array}{c}
\frac{\partial}{\partial x^{T}} \\
\vdots \\
\frac{\partial}{\partial x^{n}}
\end{array}\right)=\boldsymbol{v}^{T} \nabla \\
& \boldsymbol{v}=\left(\begin{array}{ccc}
a_{11} & \cdots & a_{1 n} \\
\vdots & \cdots & \vdots \\
a_{n 1} & \cdots & a_{n n}
\end{array}\right)\left(\begin{array}{c}
x^{1} \\
: \\
x^{n}
\end{array}\right)=A x .
\end{aligned}
$$

This $\mathfrak{g}$ has a very straightforward representation as a map $\rho: \mathfrak{g} \rightarrow \mathfrak{g l}_{n}(\boldsymbol{R})=$ End $\left(\boldsymbol{R}^{n}\right): \mathcal{L} \mapsto A$ Thus, we know that $\mathfrak{g} \cong \mathfrak{g l}_{n}(\boldsymbol{R})$, which is the Lie algebra of $G L_{n}(\boldsymbol{R})$.

Particularly, hereafter we consider the contours of objects of which the tangent or normal vector fields form linear Lie subalgebras.

Our interest is in how the infinitesimal generators, instead of the contour $M$ itself, change under a rotation. We wish at first to find the complete set of the invariants of the representation of $\mathfrak{g l}_{n}(\boldsymbol{R})$ under $S O_{n}(R)$.

In the first place, it can be noticed that when a shape, or equivalently, the generator $v$ of its Lie algebra is subjected to a rotational transform $R(\theta)$, the Lie generator becomes

$$
R(\theta) v\left(R^{-1}(\theta) x\right)=R(\theta) A R^{-1}(\theta) x
$$

i.e., the representation of the linear Lie algebra is subjected to a conjugate action. In fact, it is known that all automorphisms of $\mathfrak{s l}_{n}(\boldsymbol{R})$ (the Lie algebra of $S L_{n}(\boldsymbol{R})$ ) are adjoint actions or $A_{d}$-actions of $S L_{n}(\boldsymbol{R})$

$$
Q^{g}:=g Q g^{-1}, \quad g \in S L_{n}(R), \quad Q \in \mathfrak{s l}_{n}(R)
$$

Hereafter, we will consider a somewhat more general $A_{d}$-action, the action of the subgroup $S O_{n}(\boldsymbol{R})$ on $\mathfrak{g l}_{n}(\boldsymbol{R})$.

\section{Invariants of $2 \mathrm{D}$ linear Lie subalgebra under rotation}

We now define a linear subalgebra $\mathfrak{g}$ generated by the following basis in $2 \mathrm{D}$ case. Let $\left\{e_{1}, e_{2}, e_{3}, e_{4}\right\}$ as basis generators.

$$
e_{1}=x^{1} \frac{\partial}{\partial x^{1}}, \quad e_{2}=x^{2} \frac{\partial}{\partial x^{2}}, \quad e_{3}=x^{2} \frac{\partial}{\partial x^{1}}, \quad e_{4}=x^{1} \frac{\partial}{\partial x^{2}} .
$$

It is easy to check that the above algebra $\mathfrak{g}$ is a Lie algebra since it is closed under Lie bracket. i.e. $\forall e_{i}, e_{j} \in \mathfrak{g}, \quad\left[e_{i}, \boldsymbol{e}_{j}\right] \in \mathfrak{g}$. Thus $\mathfrak{g} \cong \mathfrak{g l}_{2}(\boldsymbol{R})$ 
An arbitrary infinitesimal generator in $\mathfrak{g}$ can be expressed as

$$
\mathcal{L}=\left(a x^{1}+b x^{2}, c x^{1}+d x^{2}\right)\left(\frac{\frac{\partial}{\partial \vec{g}^{1}}}{\frac{\partial x^{2}}{2}}\right) \boldsymbol{v}=A \boldsymbol{x}
$$

If we choose another basis of $\mathfrak{g l}_{2}(\boldsymbol{R})$ as

$$
I=\left(\begin{array}{ll}
1 & 0 \\
0 & 1
\end{array}\right), \quad H=\left(\begin{array}{cc}
1 & 0 \\
0 & -1
\end{array}\right) J=\left(\begin{array}{cc}
0 & 1 \\
-1 & 0
\end{array}\right), \quad K=\left(\begin{array}{ll}
0 & 1 \\
1 & 0
\end{array}\right)
$$

where $I$ spans the radical part of $\mathfrak{g l}_{2}(\boldsymbol{R})$ and $H, J, K$ span the semi-simple part. Then, an arbitrary representation $A$ of $\mathcal{L}$ splits as,

$$
A=\frac{a+d}{2} I+\frac{a-d}{2} H+\frac{b+c}{2} K+\frac{b-c}{2} J .
$$

Theorem 1. For

$$
\forall A=\left(\begin{array}{ll}
a & b \\
c & d
\end{array}\right) \in \mathfrak{g l}_{2}(\boldsymbol{R}),
$$

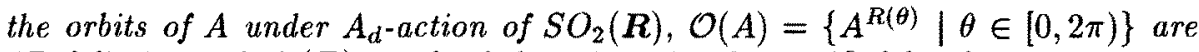
$1 D$ foliations of $\mathfrak{g l}_{2}(\boldsymbol{R})$, each of them is uniquely specified by three invariants: $\operatorname{inv}\left(A^{R(\theta)}\right)=\{\gamma, \tau, \delta\}$

$$
A^{R(\theta)}=\frac{1}{2}\left(\begin{array}{cc}
\tau & \gamma \\
-\gamma & \tau
\end{array}\right)+\frac{\sqrt{\gamma^{2}+\tau^{2}-4 \delta}}{2} R\left(2 \theta^{\prime}\right) H
$$

where $\gamma=b-c, \tau=a+d, \delta=a d-b c, \theta^{\prime} \in[0, \pi)$.

Proof: First, we know that the orbit $S \mathrm{O}_{2} \backslash \mathfrak{g l}_{2}() \boldsymbol{R}$ has dimension $\operatorname{dim} \mathcal{O}(A)=$ $\operatorname{dim} \mathrm{SO}_{2}(\boldsymbol{R})-\operatorname{dim}\left(\operatorname{center}\left(\mathrm{SO}_{2}(\boldsymbol{R})\right)\right)=1$, since center $\left(\mathrm{SO}_{2}(\boldsymbol{R})\right)$ is trivial.

As we know, $R \in S_{2}(R)$ is spanned by $I$ and $J, R(\theta)=\cos \theta I+\sin \theta J \in$ $\{I, J\}$. The subalgebra spanned by both $I$ and $J$, therefore $\{I, J\}$ are $A_{d^{-}}$ invariant. Thus, we have $(\tau I+\gamma J)$ as the rotationally invariant component. While $H$ and $K$ are not $A_{d}$-invariant, the submodule $\{H, K\}$ is closed under the $A_{d}$-action. i.e. $H^{R(\theta)}=R(2 \theta) H, \quad K^{R(\theta)}=R(2 \theta) K$

Thus, $\quad A^{R(\theta)}=\frac{\tau}{2} I+\frac{\gamma}{2} J+R(2 \theta)\left(\frac{a-d}{2} H+\frac{b+c}{2} K\right)$

Note that $P:=\frac{a-d}{2} H+\frac{b+c}{2} K \in O_{2}(\boldsymbol{R})$. (In fact, skew-symmetric subalgebra $\{I, J\}$ and symmetric submodule $\{H, K\}$ are the two disconnected components of $O_{2}(R)$.) There exists a $\theta_{0} \in[0,2 \pi)$ such that

$$
R(2 \theta) P=\frac{\sqrt{\tau^{2}+\gamma^{2}-4 \delta}}{2} R\left(2 \theta+\theta_{0}\right) H .
$$

From the above equation, $\theta_{0}$ is only a constant translation on the orbit, so we can take a new variable $\theta^{\prime}=\theta+\theta_{0} / 2$, which proves the theorem.

QED

Remark 1: The relative Euler angle $\theta^{\prime}$ between the observed object and the standard one in database can be easily calculated and used for pose estimation. Remark 2: Theorem 1 and 2 below can be easily extended to Affine Lie algebras to accomodate translations. 


\section{Invariants of 3D linear Lie subalgebra under rotation}

Now we proceed to consider 3D objects or spatial curves and surfaces under action of a 3D rotation. For spatial curves, we assume that their tangential or normal Lie algebra is the linear subalgebra $\mathfrak{g}$. For spatial surfaces, we can also consider their tangential Lie algebras or tangent vector field, which are planar or 2D subalgebra. More conveniently, we may instead look at the normal vector fields of their tangent spaces, which are 1D subalgebras. Thus, we assume also that the linear subalgebra $\mathfrak{g}$ is generated by following basis. $\left\{x^{i} \frac{\partial}{\partial x^{j}} i, j=1,2,3\right\}$ The above algebra $\mathfrak{g}$ can be checked to be closed under Lie bracket. Then an arbitrary infinitesimal generator in $\mathfrak{g}$ can be expressed as

$$
\mathcal{L}=\boldsymbol{v}^{T} \nabla, \quad \boldsymbol{v}=A \boldsymbol{x} \quad A \in \mathfrak{g l}_{3}(\boldsymbol{R})
$$

$\mathfrak{g}$ has representation as a map $\rho: \mathfrak{g} \rightarrow \mathfrak{g l}_{3}(\boldsymbol{R})=\operatorname{End}\left(\boldsymbol{R}^{3}\right): \mathcal{L} \mapsto \mathcal{A}$.

Theorem 2. For $\forall A \in \mathfrak{g l}_{3}(\boldsymbol{R})$, its orbit under the $A_{d}$ action of $S_{3}(\boldsymbol{R})$ is a $3 D$ foliation of $\mathfrak{g l}_{\mathbf{3}}(\boldsymbol{R})$,

$$
\begin{aligned}
& \mathcal{O}(A)=\left\{A^{R}\right\}=\left\{R^{\prime} \Lambda R^{0}\left(R^{\prime}\right)^{T} \quad \mid \quad \forall R \in S O_{3}(R)\right\} \\
& \text { here } \quad S O_{3}(R) \ni R^{0}=R^{0}\left(\theta^{0}, \phi^{0}, \psi^{0}\right)=V U^{T} \text {, } \\
& S O_{3}(R) \ni R^{\prime}=R^{\prime}\left(\theta^{\prime}, \phi^{\prime}, \psi^{\prime}\right)=U R^{T}
\end{aligned}
$$

where $U, V$ and $\Lambda=\operatorname{diag}\left\{\lambda_{1}, \lambda_{2}, \lambda_{3}\right\}$ denotes the singular decomposition of $A^{R}=$ $U^{T} \Lambda V$ with signs of the singular-values adjusted such that $U, V \in S_{3}(\boldsymbol{R})$. Here $\theta^{0}, \phi^{0}, \psi^{0}$ are Euler's angles of $R^{0}=V U^{T} \in S O_{3}(\boldsymbol{R})$. Each orbit is determined uniquely by the complete set of invariants:

$$
\operatorname{inv}\left(A^{R}\right)=\left\{\lambda_{1}, \lambda_{2}, \lambda_{3}, \theta^{0}, \phi^{0}, \psi^{0}\right\} .
$$

Proof: A generic orbit of $S O_{3}(\boldsymbol{R}) \backslash \mathfrak{g l}_{3}(\boldsymbol{R})$ has dimension $\operatorname{dim} \mathcal{O}(A)=\operatorname{dim} S \mathrm{O}_{3}(\boldsymbol{R})$ $\operatorname{dim}\left(\operatorname{center}\left(\mathrm{SO}_{3}(\boldsymbol{R})\right)\right)=3$. Or one can obtain the dimension of the orbit $\mathcal{O}(A)$ by calculation of the dimension of the tangent space of the orbit $T_{A} \mathcal{O}(A)=$ $\left\{[A, B] \mid \forall B \in \mathfrak{s o}_{3}\right\}$. Thus, the orbits of the adjoint action of $S O_{3}(\boldsymbol{R})$ are 3D foliation of $9 \mathrm{D}$ space of $\mathfrak{g l}_{3}(\boldsymbol{R})$, thus each of them is uniquely specified by 6 invariants.(See Fig.1)

For $\forall A \in \mathfrak{g l}_{n}(\boldsymbol{R})$, we have the singular decomposition $A=U^{T} \Lambda V$, where $\left\{\lambda_{i}\right\}$ are $A$ 's singular values with signs adjusted such that $U, V$ are in $S O_{n}(\boldsymbol{R})$. Thus, the representation of the considered Lie algebra under $R \in S O_{n}(R)$ becomes $A^{R}=R U^{T} \Lambda V R^{T}=\left(R^{\prime}\right)^{T} K R^{\prime}$ Here, $K=A V U^{T}$. Since the singular decomposition of any points on the orbit $A^{R}=\left(U^{\prime}\right)^{T} \Lambda V^{\prime}=R U^{T} \Lambda V R^{T}$, where $U^{\prime}=U R^{T}, V^{\prime}=V R^{T}$. It is easy to see that $R\left(\theta^{0}, \phi^{0}, \psi^{0}\right)=V U^{T}=V^{\prime}\left(U^{\prime}\right)^{T}$ thus $K$ is invariant on the whole orbit of $A$ in $\mathfrak{g l}_{3}(\boldsymbol{R})$.

Since such $K$ uniquely determines the orbit and is 6 dimension, the set $\left\{\lambda_{1}, \lambda_{2}, \lambda_{3}, \theta^{0}, \phi^{0}, \psi^{0}\right\}$ is the complete set of invariants of the foliations. QED 


\section{Comparison with algebraic shapes}

Weconsider the normal Lie algebra of a 3D algebraic surface defined by the following polynomials. (2D curves can be dealt with similarly.) The normal algebras of $3 \mathrm{D}$ algebraic curves can also be obtained in the same way.

$$
\begin{gathered}
F(x, y, z)=a x^{2}+b y^{2}+c z^{2}+d x y+e x z+f y z+g x+h y+i z+j=0 \\
\nabla F=\left(\begin{array}{ccc}
2 a & d & e \\
d & 2 b & f \\
e & f & 2 c
\end{array}\right)\left(\begin{array}{l}
x \\
y \\
z
\end{array}\right)+\left(\begin{array}{c}
g \\
h \\
i
\end{array}\right)=A x+b
\end{gathered}
$$

Thus, one can see that algebraic shapes can be represented by Affine Lie algebras. Notice that using normal algebras, we always have the representation with a symmetric matrix $A$.

In table 1, we take a comparison between the method using algebraic invariants and the proposed method. The linear Lie subalgebra, even as a simple kind of subalgebra, is able to represent all quadratic algebraic shapes, and far more rich classes of nonalgebraic or nonpolynomial curves and surfaces as well.

\begin{tabular}{|c|c|c|}
\hline Dimension of Shapes & Algebraic shapes & Linear Lie algebra \\
\hline $2 \mathrm{D}$ & 2 & 3 \\
\hline $3 \mathrm{D}$ & 3 & 6 \\
\hline
\end{tabular}

Table 1. Comparison of number of Invariants

\section{Implementation and Simulation}

The implementation of the proposed scheme is also easy in practice, by solving linear equations using the observed data of three (in 2D case) and four (in 3D case) or more points on the object surface.

Here we only show a recognition procedure of $3 \mathrm{D}$ objects.

Step 1. Take normal unit vectors of $3 D$ contour of the object from more than three points.

$$
\left\{\left(x_{k}, y_{k}, z_{k}\right),\left(\alpha_{k}, \beta_{k}, \gamma_{k}\right)\right\}, \quad k=1, \cdots, 4
$$

Step 2. We assume also dilation invariance so that $a$ can be fixed to 1 . Then build matrix equations in elements of a representation $\mathrm{A}$.

$$
\left(\begin{array}{l}
\alpha_{k} \\
\beta_{k} \\
\gamma_{k}
\end{array}\right)=\lambda_{k}\left(\begin{array}{lll}
1 & b & c \\
d & e & f \\
g & h & i
\end{array}\right)\left(\begin{array}{l}
x_{k} \\
y_{k} \\
z_{k}
\end{array}\right)
$$

where $\lambda_{k}$ 's are scaling coefficients as before. 
Step 3. Delete $\lambda_{k}$ from the above simultaneous equation to obtain linear equations:

$$
\left(\begin{array}{c}
\beta_{1} x_{1} \\
\beta_{2} x_{2} \\
\beta_{3} x_{3} \\
\beta_{4} x_{4} \\
\gamma_{1} x_{1} \\
\gamma_{2} x_{2} \\
\gamma_{3} x_{3} \\
\gamma_{4} x_{4}
\end{array}\right)=\left(\begin{array}{cccccccc}
-\beta_{1} y_{1} & \beta_{1} z_{1} & \alpha_{1} x_{1} & \alpha_{1} y_{1} & \alpha_{1} z_{1} & 0 & 0 & 0 \\
-\beta_{2} y_{2} & \beta_{2} z_{2} & \alpha_{2} x_{2} & \alpha_{2} y_{2} & \alpha_{2} z_{2} & 0 & 0 & 0 \\
-\beta_{3} y_{3} & \beta_{3} z_{3} & \alpha_{3} x_{3} & \alpha_{3} y_{3} & \alpha_{3} z_{3} & 0 & 0 & 0 \\
-\beta_{4} y_{4} & \beta_{4} z_{4} & \alpha_{4} x_{4} & \alpha_{4} y_{4} & \alpha_{4} z_{4} & 0 & 0 & 0 \\
-\gamma_{1} y_{1} & -\gamma_{1} z_{1} & 0 & 0 & 0 & \alpha_{1} x_{1} & \alpha_{1} y_{1} & \alpha_{1} z_{1} \\
-\gamma_{2} y_{2} & -\gamma_{2} z_{2} & 0 & 0 & 0 & \alpha_{2} x_{2} & \alpha_{2} y_{2} & \alpha_{2} z_{2} \\
-\gamma_{3} y_{3} & -\gamma_{3} z_{3} & 0 & 0 & 0 & \alpha_{3} x_{3} & \alpha_{3} y_{3} & \alpha_{3} z_{3} \\
-\gamma_{4} y_{4} & -\gamma_{4} z_{4} & 0 & 0 & 0 & \alpha_{4} x_{4} & \alpha_{4} y_{4} & \alpha_{4} z_{4}
\end{array}\right)\left(\begin{array}{c}
b \\
c \\
d \\
e \\
f \\
g \\
h \\
i
\end{array}\right)
$$

Solve the equations to estimate the representation $A$.

Step 4. Use theorem 2 to calculate the set of invariants from derived in Step 3, and do feature matching.

Remark 1 The two or three points on the 2D or 3D contours can be choosed almost arbitrarily, e.g., from some small or observable part of the object under occlusion. As an advantage of the method, the global shape can still be recognized from these local and partial features.

Remark 2: In order to gain numerical stability, one may use Hugh transformation to estimate normal vectors and the least square fitting in solving the linear equations.

Remark 3: The shape represention by the linear Lie algebra can also be used as patches for more complicated objects. In that case, the number of patches can be reduced and segmentation is also easy. One can divide the shape into segmentations within which the variations of invariants are under certain prechosen threshold.

Remark 4: Another problems remained is to investigate the class of shapes which are representable by the linear Lie algebras among all 2D and 3D shapes. It seems to be theoretically difficult. So we tried this by simulation. Fig. $2 \sim 9$ shown a part of the results for 3D case. When $A$ is symmetric, one will obtain algebraic surfaces such as ellipsoid in Fig.2, hyperboloid in Fig.3 and elliptic cone in Fig.4 etc. For nonsymmetric $A$, a large variety of non-algebraic surfaces can be derived e.g. as shown in Fig. 5 9 .

\section{References}

1. Mundy, J.L. and Zisserman A.(ed) Geometric invariance in computer vision MIT Press, Cambridge Ma. 1982.

2. D. Forsyth , J. L. Mundy, A. Zisserman "Invariant descriptors for 3-D object recognition and pose", IEEE Trans.. PAMI, vol13, No. 10, 971-991, October (1991)

3. T. Moons, E. J. Pauwels, L. J. Van Gool and A. Oostserlinck, "Foundation of semidifferential Invariants" Int. Journal of Computer Vision, 14, 25-47 (1995)

4. E. J. Pauwels, T. Moons, L. J. Van Gool, P. Kempenaers and A. Oostserlinck, "Recognition of planar shapes under affine distortion" Int. Journal of Computer Vision, 14, 48-51 (1995) 


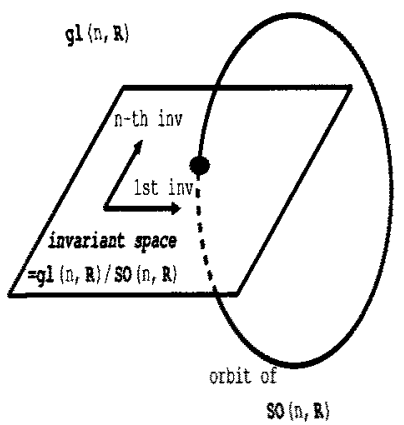

Fig. 1. Invariant space

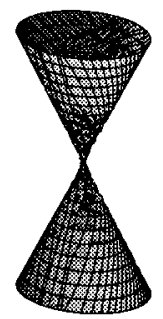

Fig. 4. Elliptic cone

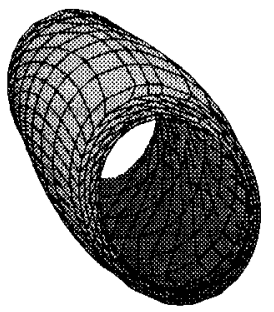

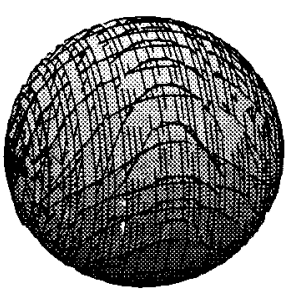

Fig. 2. Ellipsoid

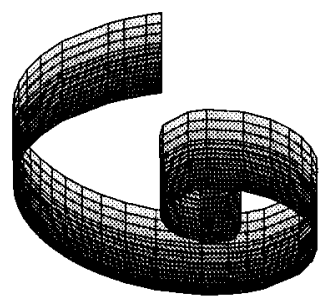

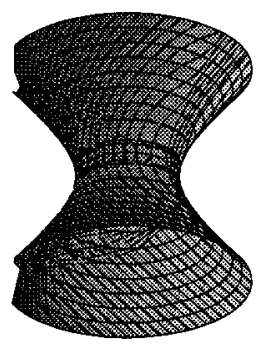

Fig. 3. Hyperboloid

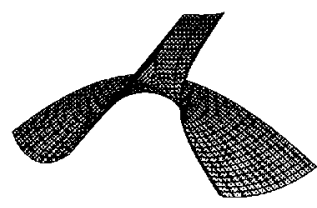

Fig. 5. Nonalgebraic shape1 Fig. 6. Nonalgebraic shape2
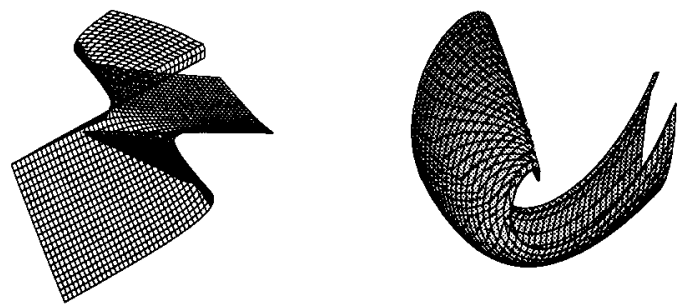

Fig. 7. Nonalgebraic shape3 Fig. 8. Nonalgebraic shape4 Fig. 9. Nonalgebraic shape5 